Technological University Dublin

DÜBLIN

ARROW@TU Dublin

2008-01-01

\title{
A Visual Indication of Environmental Humidity using a Color Changing Hologram Recorded in a Self-developing Photopolymer
}

Izabela Naydenova

Technological University Dublin, izabela.naydenova@tudublin.ie

Raghavendra Jallapuram

Technological University Dublin, mrutic@gmail.com

Vincent Toal

Technological University Dublin, vincent.toal@tudublin.ie

See next page for additional authors

Follow this and additional works at: https://arrow.tudublin.ie/scschphyart

Part of the Physics Commons

\section{Recommended Citation}

Naydenova, l. et al. (2008) A Visual Indication of Environmental Humidity using a Color Changing Hologram Recorded in a Self-developing Photopolymer. Applied Physics Letters, 92, 031009. doi:10.1063/ 1.2837454

This Article is brought to you for free and open access by the School of Physics \& Clinical \& Optometric Science at ARROW@TU Dublin. It has been accepted for inclusion in Articles by an authorized administrator of ARROW@TU Dublin. For more information, please contact arrow.admin@tudublin.ie, aisling.coyne@tudublin.ie, gerard.connolly@tudublin.ie.

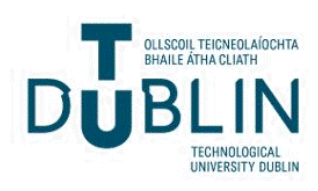




\section{Authors}

Izabela Naydenova, Raghavendra Jallapuram, Vincent Toal, and Suzanne Martin

This article is available at ARROW@TU Dublin: https://arrow.tudublin.ie/scschphyart/3 


\title{
A visual indication of environmental humidity using a color changing hologram recorded in a self-developing photopolymer
}

\author{
Izabela Naydenova, ${ }^{\text {a) }}$ Raghavendra Jallapuram, Vincent Toal, and Suzanne Martin \\ Centre for Industrial and Engineering Optics, School of Physics, Dublin Institute of Technology, Kevin Street, \\ Dublin 8, Ireland
}

(Received 15 November 2007; accepted 21 December 2007; published online 23 January 2008)

\begin{abstract}
A reflection hologram for visual indication of environmental humidity has been studied. The hologram is recorded in a self-developing photopolymer and changes color when exposed to a change in humidity and is fully reversible. The range of color change, reversibility, and the response time of the hologram have been studied in a controlled humidity environment. Fully reversible holograms with response times from few seconds to tens of minutes have been designed. Extremely sensitive bright visual humidity indicators, capable of dramatic color change within a few seconds of breathing on them are demonstrated. (C) 2008 American Institute of Physics.
\end{abstract}

[DOI: $10.1063 / 1.2837454]$

Relative humidity $(\mathrm{RH})$ is of special interest for many industrial and domestic applications. In order to improve product quality, monitoring of the relative humidity during many industrial processes is required. Usually, the relative humidity is measured and controlled by humidity meters that detect change in a physical property of a thin film, such as capacitance, resistivity or thermal conductivity. These humidity control systems require a power source, and are relatively complex and expensive. It is also often important to monitor relative humidity during product transport and storage utilizing simple and inexpensive indicator devices incorporated into packaging. An example of such a device is a card containing spots of silica gel that indicate a change in relative humidity by a color change. However, they often have a slow response time and limited accuracy. Disposable holographic sensors that change their properties when exposed to different environmental conditions could provide an easily interpreted, visual response with an added advantage of faster response time and better accuracy.

The sensitivity of some holographic recording materials to humidity has been known for many years. ${ }^{1-3}$ In silver halide materials for instance it has been used to achieve color control in reflection holograms. ${ }^{1}$ Special care to avoid exposure to high humidity is required for other materials ${ }^{2,3}$ as it destroys the recorded hologram. The design of a hologrambased humidity sensor requires a unique combination of properties of the holographic recording material-high sensitivity to humidity and accompanying resistance to damage by humidity. To achieve this combination of properties appears to be very challenging as no successful fabrication of such a holographic device has been reported so far. A humidity sensor using a wavelength-dependent holographic filter with fiber optic links was reported by Spooncer et al. in Ref. 4. The sensor medium, gelatin, was characterized by a strong hysteresis in humidity response, temperature dependence, and variable sensitivity and reported as unsuitable for use as a sensor. Although not designed specifically for the detection of humidity, sensors operating on a similar principle have also been demonstrated in Ref. 5. In the present paper, to the best of our knowledge we report for the first time the suc-

${ }^{a)}$ Electronic mail: izabela.naydenova@dit.ie. cessful fabrication and characterization of a visible, highly sensitive, and fully reversible humidity sensitive reflection hologram recorded in a self-processing photopolymer.

In general, a hologram is produced in a photosensitive material by exposure to an interference pattern produced by two coherent light beams. The spatial variation in light intensity or state of polarization is recorded in the material as a variation in refractive index, absorption or thickness. In the most general case, the recorded pattern is a diffraction grating that produces diffracted light when illuminated with light of appropriate spectral or polarization characteristics and in the appropriate direction. The properties of the diffracted light-intensity, wavelength, phase or state of polarization depend on the properties of the recorded diffraction pattern and are useful for the design of holographic sensors. A significant advantage of such a sensor would be the possibility of a visual indication when exposed to the environment to which it is sensitive. The availability of a large variety of holograms and holographic materials makes the design of holographic sensors a very flexible process. The present paper focuses on the humidity sensing properties of a reflection phase hologram recorded in an acrylamide-based photopolymer.

Figure 1 illustrates the basic principle of the humidity sensitive hologram. The high spectral selectivity of the reflection holograms ${ }^{6}$ means that light of a specific color is diffracted when the hologram is illuminated with white light. The color observed depends on the holographic fringe spacing $\Lambda$ given by

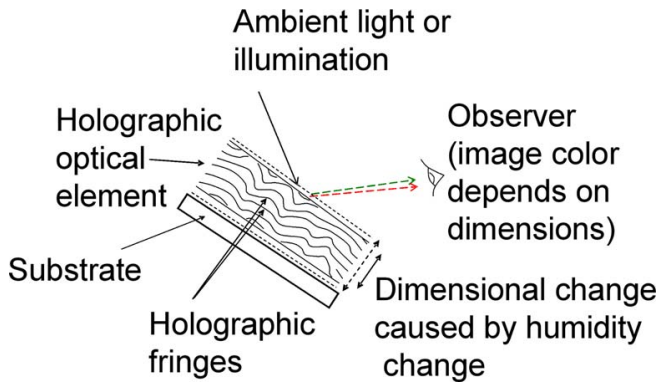

FIG. 1. (Color online) Principle of operation of the humidity sensitive reflection hologram. 


$$
\Lambda=\lambda /\left(2 n_{0} \sin \theta\right),
$$

where $\theta$ is half of the angle between the recording beams inside the recording medium, $\lambda$ is wavelength of the recording light and $n_{0}$ is the refractive index of the recording material. According to Eq. (1), there are two possible mechanisms for change in the reconstructed or diffracted wavelength (and, thus, in the observed color) for a hologram that is disposed in a medium that can easily absorb or release water as the relative humidity of the environment changes. Firstly, dimensional changes of the recording medium occur due to the ease with which the material swells or shrinks, leading to change in the fringe spacing. Secondly, if there is a change in the overall refractive index of the medium due to the absorption of moisture, the optical path length between recorded fringes will change. The relative contributions of the two mechanisms will depend mainly on the properties of the recording medium. The ultimate effect is a change of the observed color of the diffracted light as a result of change of the environmental humidity.

The material used in this study is a self-processing acrylamide-based photopolymer developed at the Centre for Industrial and Engineering Optics, Dublin Institute of Technology. ${ }^{7-10}$ The humidity indicators were fabricated using an optimized photopolymer composition for recording in reflection mode ${ }^{11}$ containing $0.8 \mathrm{~g}$ acrylamide, $0.25 \mathrm{~g}$ $N, N^{\prime}$ methylene bisacrylamide, $1.5 \mathrm{ml}$ triethanolamine, $17.5 \mathrm{ml}$ of $10 \% \mathrm{w} / \mathrm{v}$ polyvinylalcohol stock solution, and $3 \mathrm{ml}$ of $0.11 \% w / v$ of Erythrosin B stock solution. Layers with thicknesses of $30-180 \mu \mathrm{m}$ were obtained by varying the volume of the photopolymer solution gravity settled on a $50 \times 50 \mathrm{~mm}^{2}$ glass slide between 0.5 and $2 \mathrm{ml}$. The samples were dried for $24 \mathrm{~h}$. The frequency doubled output from a $\mathrm{NdYVO}_{4}$ laser was used to record the reflection holograms at $532 \mathrm{~nm}$ wavelength using a standard two beam geometry, allowing for control and equalization of the intensities of the two beams. The total exposure required to achieve $30 \%$ diffraction efficiency did not exceed $100 \mathrm{~mJ} / \mathrm{cm}^{2}$. Humidity indicators incorporating a holographic image of an object were recorded as Denisyuk type holograms.

A controlled environment chamber with humidity control system (Electro-Tech systems model 503-20) was used to characterize the reflection holograms. The relative humidity in the chamber could be maintained at a set point in the range of 5\%-100\% RH with accuracy better than $\pm 1 \% \mathrm{RH}$. The optical test setup was assembled inside the humidity chamber. Light from an AvaLight-HAL-S light source was fiber guided into the humidity chamber and then collimated and used to probe the holograms. The diffracted light from the hologram was coupled into a second fiber by a lens and guided to a spectral analyzer, AvaSpec-2048. To obtain the calibration curve for a specific hologram, the relationship between the wavelength peak in the spectral response of the hologram and the relative humidity in the chamber was determined. The response time of the hologram was measured by recording the change of the spectral peak position with time after achieving a preset humidity. The change in the peak position during the time needed to achieve the preset humidity for layers with thickness above $30 \mu \mathrm{m}$ was negligible. The response time was also studied by recording the holographic images with a digital camera Sony Cybershot DSC300 at different times after the change in the relative humidity was introduced.

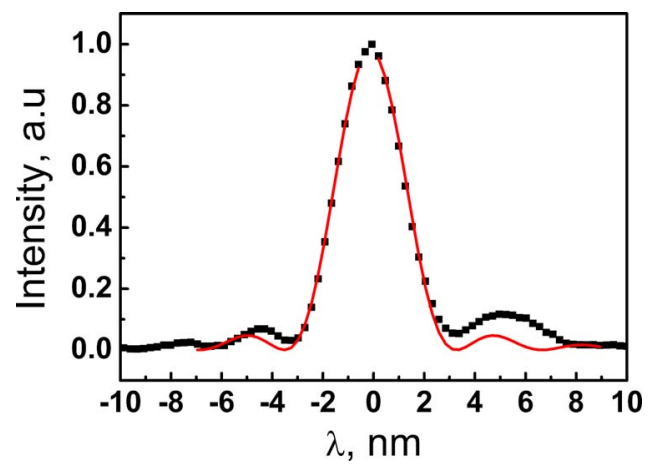

FIG. 2. (Color online) Spectral response of a hologram recorded in a $30 \mu \mathrm{m}$ thick layer (squares) and theoretical fit (red line).

The typical spectral response of a reflection holographic grating recorded in a $30 \pm 3 \mu \mathrm{m}$ thick photopolymer layer is presented in Fig. 2. The thickness of the hologram, obtained by fit of the experimental data with the well known function $^{12}$ describing the wavelength selectivity of a reflection hologram, was $28 \mu \mathrm{m}$, in good agreement with the results from independent measurement of the layer thickness. As expected such a holographic optical element ${ }^{6,12}$ is highly selective with respect to the wavelength of the diffracted light. The full width at the half maximum is $2.8 \mathrm{~nm}$. This explains monochromatic appearance of the reconstructed image when the recorded object is relatively flat (Fig. 3) and there is a limited range of spatial frequencies in the recorded hologram.

To calibrate the humidity response of the hologram, the change of the spectral peak position was determined for different relative humidities. A characteristic example of the humidity response of a reflection holographic grating recorded in $30 \mu \mathrm{m}$ layer is shown in Fig. 4. It can be seen that when the relative humidity changes from 5\% to $80 \%$ at $23{ }^{\circ} \mathrm{C}$ the position of the peak in the hologram's spectral response increases by $130 \mathrm{~nm}$. To identify the mechanism causing this effect, one can differentiate Eq. (1) and, assuming that the average refractive index is unchanged, one can calculate that the observed change in the reconstructed wavelength can be produced by approximately $10 \%$ change in the fringe spacing and consequently in the volume of the hologram. Such a change in volume can be easily expected taking into account the highly hygroscopic nature of the polyvinyl alcohol binder and the polyacrylamide formed during the recording of the hologram. Alternatively using the same equation and, assuming that the fringe spacing remains constant, the change in the average refractive index required to cause a $130 \mathrm{~nm}$ shift in the diffracted wavelength is in order of $0.3-0.4$. This value is extremely high and suggests that the first mechanism is mainly responsible for the observed color change. Further studies using a white light interferometer to

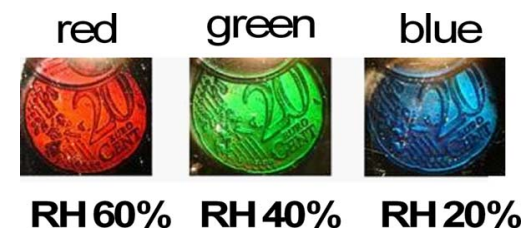

FIG. 3. (Color online) Color appearance of a hologram exposed to different

humidity levels. 


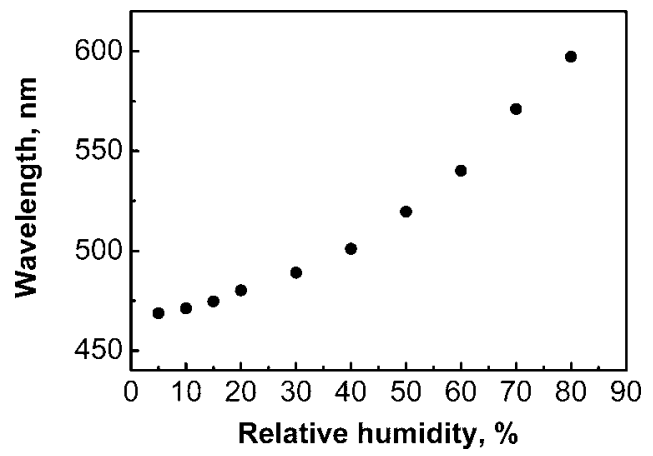

FIG. 4. Humidity response of a reflection hologram recorded in $30 \mu \mathrm{m}$ thick photopolymer layer.

measure the dimensional change will determine the exact contribution of each process.

Initial studies in a controlled humidity and temperature environment show no substantial temperature dependence of the response of the hologram. When the temperature changes from 23 to $50{ }^{\circ} \mathrm{C}$ at constant humidity of $30 \%$, the spectral peak shifts by $1.6 \mathrm{~nm}$. More detailed studies on the temperature dependence of the humidity response are in progress.

In order to study the reversibility of the humidity response, the hologram was exposed to seven cycles of change in relative humidity, from $20 \%$ to $80 \% \mathrm{RH}$. The position of the spectral peak was repeatable within $1 \mathrm{~nm}$, a change in the visual appearance of the hologram that is too small to be detected. Further studies involving larger numbers of cycles are underway.

The response times of samples recorded in photopolymer layers with different thicknesses were also studied. It was observed that the thickness of the layers has a significant influence on the time required to achieve the final color appearance at specific relative humidity. For $30 \mu \mathrm{m}$ thick layer, it took $10 \mathrm{~min}$ to reach a fixed color when the humidity was changed from $50 \%$ to $60 \% \mathrm{RH}$ and for the $90 \mu \mathrm{m}$ thick layer as long as $50 \mathrm{~min}$ was necessary. After optimization of the thickness of the recording layer, a response time of as fast as a few seconds was achieved. Pictures from a very sensitive hologram are shown in Fig. 5. The hologram changes color

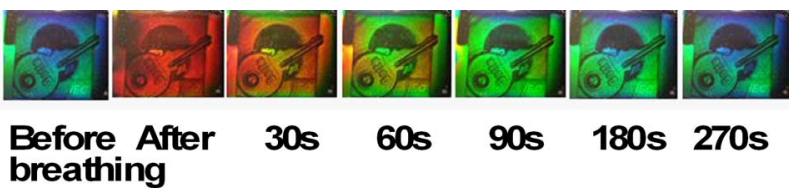

FIG. 5. (Color online) Color appearance of a reflection hologram before and after breathing on it.

in less than $30 \mathrm{~s}$ after breathing on it and the initial color appearance is restored within $5 \mathrm{~min}$.

In conclusion, we have successfully recorded a high diffraction efficiency, photopolymer based, humidity sensitive, reflection hologram, which changes color when exposed to different relative humidities, mainly due to swelling or shrinkage of the photopolymer medium. The reversibility, low dependence on temperature, and fast response times are very promising for the design of humidity indicators providing easy to interpret visual information. The response times can be controlled by the thickness of the photopolymer layer used to record the holographic humidity indicator.

The support of this research by Enterprise Ireland through its Commercialisation Fund, Technology Development phase is greatly appreciated.

${ }^{1}$ D. Wuest and R. Lakes, Appl. Opt. 30, 2363 (1991).

${ }^{2}$ G. Naik, A. Mathur, and S. Pappu, Appl. Opt. 29, 5292 (1990).

${ }^{3}$ H. L. Fielding and R. T. Ingwall, US Patent No. 4588664 (1986).

${ }^{4}$ R. C. Spooncer, F. A. Al-Ramadhan, and B. E. Jones, Int. J. Optoelectron. 7, 449 (1992).

${ }^{5}$ A. J. Marshall, J. Blyth, C. Davidson, and C. R. Lowe, Anal. Chem. 75, 4423 (2003).

${ }^{6}$ P. Hariharan, Optical Holography (Cambridge University Press, Cambridge, England, 1996).

${ }^{7}$ S. Martin, P. Leclere, Y. Renotte, V. Toal, and Y. Lion, Opt. Eng. (Bellingham) 33, 3942 (1992).

${ }^{8}$ S. Martin, C. A. Feely, and V. Toal, Appl. Opt. 36, 5757 (1997).

${ }^{9}$ I. Naydenova, R. Jallapuram, S. Martin, R. Howard, and V. Toal, Appl. Opt. 43, 2900 (2004).

${ }^{10}$ Raghavendra Jallapuram, Ph.D. thesis, Dublin Institute of Technology, 2005.

${ }^{11}$ I. Naydenova, H. Sherif, S. Martin, R. Jallapuram, and V. Toal, Patent No. WO2007060648 (2007).

${ }^{12}$ H. Kogelnik, Bell Syst. Tech. J. 48, 2909 (1969). 